- Ig M+ and Ig G+ cells accumulated mostly from the 1 st day of lactation and did not undergo morphological changes. They could migrate in early lactation and only undergo a terminal differentiation in the mammary parenchyma.

In conclusion, there seems to be a local and autonomous immune system as shown on the one hand by the presence of all the lymphoid cells involved in an immune response and on the other hand by the independent quantitative variations of blood levels.

\title{
A new porcine coronavirus in french pig herds
}

\author{
A. JESTIN, Y. LEFORBAN, P. VANNIER \\ Ministère de l'Agriculture, Direction de la Qualité, Services Vétérinaires, \\ Station de Pathologie Porcine, B.P. 9, 22440 Ploufragan
}

Since July 1986 a large number of pig herds (73\%) in Brittany have seroconverted to transmissible gastroenteritis (TGE) virus. This widespread seroconversion in the absence of clinical signs of enteric disease suggests the emergence of a new coronavirus exhibiting serological crossreactions with TGE virus. This new virus does not cause digestive, but rather respiratory disorders and might be in some herds associated with clinical signs observed in fattening pigs such as hyperthermia, anorexia and respiratory symptoms. A study was made in 6 herds exhibiting these symptoms. The currently used etiological methods (Aujeszky's disease, influenza) did not explain these disorders in 2 out of the 6 herds seroconverted to TGE virus. The aerial transmission of the virus probably explains the rapid spread. Similar widespread seroconversions to TGE virus have been reported in Denmark, Belgium and Great Britain. This infection is due to a TGE-like virus whose nature has not been established yet.

\section{Etiology, epidemiology and pathogenesis of streptococcosis with Streptococcus suis type 2 (Lancefield's group R) in the pig}

\author{
Josée VAISSAIRE ${ }^{(1)}$, J.P. GILLET ${ }^{(2)}$, C. MARCON ${ }^{(3)}$
}

(1) Laboratoire Central de Recherches Vétérinaires, Service de Bactériologie, 22, rue Pierre-Curie, B.P. 67, 94703 Maisons-Alfort Cedex.

(2) Laboratoire Central de Recherches Vétérinaires, Service d'Anatomopathologie, 22, rue Pierre-Curie, B.P. 67, 94703 Maisons-Alfort Cedex.

(3) D' Vétérinaire, "Lazuel ", 07200 Aubenas.

A study on streptococcal disease due to Streptococcus suis type 2 was conducted in about thirty pig herds. Samples (nasal and vaginal swabs) were taken from live animals exhibiting nervous disorders and from healthy carriers at the slaughterhouse (tonsils).

According to this study, the main carrier site is the tonsil. Very young animals are already nasal and mainly tonsillar carriers. Transmission of the infection occurs when animals are mixed or following various stress or immunodepressions. The carrier state is important in young and breeding animals. The disease is little observed in farrowing and multiplier herds.

Pathogenesis is similar to that of pneumococcus in human medicine. It is characterized by an angina with rhinitis and pneumonia turning in some animals into meningitis and septicaemia.

Some breeds might be more sensitive. 